\title{
FUZZY IF-THEN RULES CLASSIFIER ON ENSEMBLE DATA
}

\author{
TIEN THANH NGUYEN ${ }^{1}$, ALAN WEE-CHUNG LIEW ${ }^{1}$, CUONG TO ${ }^{1}$, XUAN CUONG PHAM ${ }^{2}$, MAI PHUONG \\ NGUYEN ${ }^{3}$
}

\author{
${ }^{1}$ School of Information and Communication Technology, Griffith University, Australia \\ ${ }^{2}$ School of Information and Communication Technology, Hanoi University of Science and Technology, Vietnam \\ ${ }^{3}$ College of Business, Massey University, New Zealand \\ E-MAIL: tienthanh.nguyen2@griffithuni.edu.au, a.liew@griffith.edu.au, c.chieuto@griffith.edu.au, \\ cuong.phamxuan.hust@gmail.com, phuongnm0590@gmail.com
}

\begin{abstract}
:
This paper introduces a novel framework in which classification method based on fuzzy IF-THEN rules is co-operated with an ensemble system. This model tackles several drawbacks. First, IF-THEN rules approaches have problem with high dimensional data since computational cost is exponential function. In this framework, rules are operated on outputs of base classifiers which have frequently lower dimension than original data. Moreover, outputs of base classifiers are scaled within the range [0, 1] so it is convenient to apply fuzzy rules directly instead of data transformation and normalization as requirement for inputs of fuzzy rules. The performance of this model was evaluated through experiments on 6 popular datasets from UCI Machine Learning Repository and comparisons with other state-of-art combining classifiers algorithms as well as other fuzzy IF-THEN rules approaches. The results show that our framework can improve the classification accuracy.
\end{abstract}

\section{Keyword:}

Ensemble method; Combining classifiers; Classifier fusion; fuzzy IF-THEN rules.

\section{Introduction}

Ensemble is a class of methods which combine models to achieve lower error rate than using a single model. The concept "model" in definition of ensemble methods is understood in a broad sense, including not only the implementation of many different learning algorithms, or the creation of more training set scheme for the same learning algorithm, but also generating generic classifiers in combination to improve efficiency of classification task.

Recent years, ensemble methods have been studied extensively, and are one of the most active research areas in supervised learning [25]. The meaning and importance of ensemble methods are very significant. Given a dataset, choosing a suitable algorithm is a non-trivial problem and we only use trial and error task as a solution. Here, there are two factors to be considered are classification accuracy and execution time. In many cases, the accuracy in classification is preferred however in instant decisions or online learning, the time factor also plays an important role. In those cases, people find choosing a learning algorithm has fast execution with acceptable accuracy level. Ensemble methods, in turn, can achieve higher accuracy than a single learning algorithm. Therefore, when we focus on accuracy for the classification system, ensemble methods can be an effective option.

On the other hand, fuzzy rule classifier by extracting knowledge from data successfully has been applied to different classification tasks, interesting numerous researchers to put their attentions on [26]. The first idea about IF-THEN rules in fuzzy classifier system is introduced by Valenzuela and Rendon [6] and then the model has been developing significantly by results of Ishibuchi [7-12], and Herrera with his partners [15, 19]. Recently, some interesting result has been proposed by Verikas [16] in which he used SOM tree to reduce search space which generated from genetic search on structure and parameters of fuzzy rules classifiers) and Soua [22] where she computed the correlation between each pair of component in feature vector, and then gathered high-correlation components in a single group, finally building fuzzy rules for each group. In general, a fuzzy IF-THEN rule is given by:

$$
\begin{gathered}
\text { Rule : If } X_{i 1} \in L_{1} \text { and } X_{i 2} \in L_{2} \text { and...and } X_{i D} \in L_{D} \\
\text { then } X_{i} \in \text { Class } j \text { with } C F_{j}
\end{gathered}
$$


in which $X_{i}=\left(X_{i 1}, \ldots, X_{i D}\right)$ is D-vector of observations of dataset, $L_{i}$ is an antecedent fuzzy set and $C F_{j}$ is called rule weight (i.e., certainly factor) within $[0,1]$.

A problem with fuzzy IF-THEN rules is time-consuming with high dimensional data since if we have a D-dimension dataset and $|L|$ antecedent fuzzy set, finally, the total number of rules in rule set is $|L|^{D}$. The obstruction here is that number of rules increases exponentially if number of components in original data is large. Moreover, it is needed to transform and normalize for each component in feature vector or build particular antecedent fuzzy set for each of them. Consequently, it is time consuming process and not convenient to implement in application. It inspires us to have further investigation to overcome by propose a strategy by combining fuzzy IF-THEN rules with ensemble system.

The rest of this paper is organized as the literature review on both several state-of-art combining classifiers algorithms and significant approaches related to fuzzy rules is shown at the $2^{\text {nd }}$ section. At the $3^{\text {rd }}$ sections, the novel framework is proposed and evaluated on 6 popular UCI datasets. Finally, we summarize interesting points of the model and propose several developments in the near future.

\section{Literature Review}

There are many combining algorithms proposed in which algorithms based on Stacking approach are the most popular (Ting [2]). In this model, the training set is divided into nearly equal non-overlapped B parts. One part plays the role of test set in turn and the others as training set so all observations in the original dataset will be tested once. The outputs of Stacking are posterior probabilities that observations belong to a class according to each classifier. Posterior probabilities of all observations are gathered in a group called meta-data or Level1 data to distinguish it from Level0 data which is the original data.

To apply Stacking to ensemble of classifiers, Ting [2] proposed Multiple Response Linear Regression algorithm (MLR) to combine posterior probabilities from Level1 data of each observation based on sum of weights calculated from $\mathrm{K}$ linear regression functions. Kuncheva [1] applied fuzzy relation to find the relationship between posterior probability and Decision Template for each class computed on posterior probability of observations and its true class label. She also detailed eleven measurements between two fuzzy relations [1] to predict class label of observations of interest. Metz [4] combined Stacking, Correspondence Analysis and $\mathrm{K}$ Nearest Neighbor (KNN) in a single algorithm called SCANN. His idea is using Correspondence Analysis to analyses relationship between rows (include all observations) and columns (include outputs of Stacking in Crisp Label type [1] and true label of each observation) to form the new representation of outputs from base classifiers. After that, KNN is applied to that representation to have prediction for unlabeled observations.

Moreover, one of the most popular and simple combining classifiers algorithms was introduced by Kittler [3]. He presented six fixed combining rules namely Sum, Product, Vote, Min, Max and Average. In our knowledge, Vote and Sum are the most common and successfully implemented combining rules.

In addition, we also conduct the review on fuzzy IF-THEN rules for classification task by mentioning about 4 steps in building fuzzy classification system.

Step1 (Building membership functions): This step is also called fuzzy discretization in which membership function is introduced for each component of feature vector. Ishibuchi [7] proposed fixed triangular 14 functions (Figure 1) plus a special “don't care” function (denoted as L0 with membership degree is 1 for all values of a variable) for fuzzy classification task. Meanwhile, Alcalá-Fdez [19] introduced an approach to learn these functions by context by applying Genetic Algorithm to building chromosome corresponding with each membership function. Based on value of related chromosome, the function will be moved to left or right to obtain adaptive positions. Casillas [15] built rich functions by changing shape of them from original one with the linguistic hedges "very" and "more or less". Recently, Soua [22] used trapezoidal form for membership after gathering attributes based on correlation between them.

Step2 (Rule Generation and Reduction): In this step, based on membership functions, fuzzy rule set is generated on attributes of feature vector. Ishibuchi $[8,12]$ suggested using only several antecedent conditions with high dimensional data and deleting un-useful rules which have zero rule weight.

Step3 (Rule Selection): The goal of this task is continuing to reduce number of rules in rule set so as to save computational cost and boost performance of system. Ishibuchi [8] evaluated 5 Heuristic Criteria (support, confidence, product criterion, SLAVE 

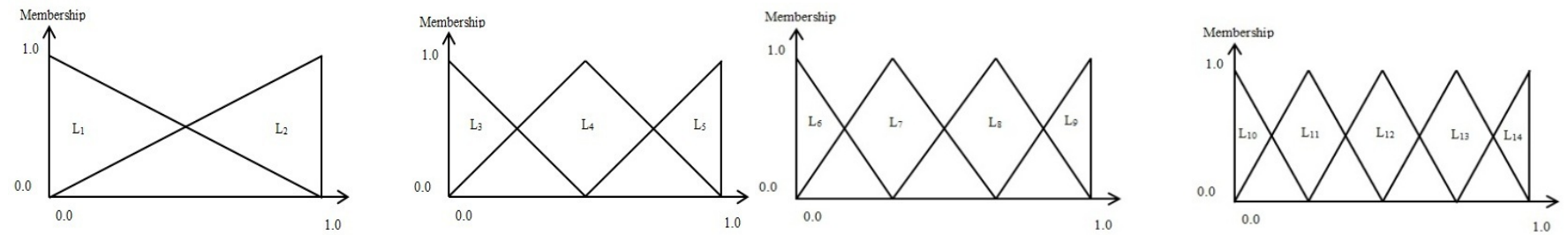

Figure1. Classical membership function

[20] and Castro [21]) for fuzzy rule selection to find which is suitable for a particular data set. Mansoori [14] introduced SGERD algorithm that select a strategy due to a novel weight function and define a threshold depended on length of rule.

Step4 (Classification based on Rule set): Level1 of all observations in test set is generated and classified due to optimal Rule set selected in Step3. Ishibuchi introduces two strategies namely Single Winner and Vote [9]. In the former, observation in test set is predicted with class label associated rules by:

$$
X \text { Test } \in \text { class } j^{*} \text { if } j^{*}=\arg \max _{j}\left(\mu_{R_{j}}(X T e s t) \times C F_{j}\right)
$$

in which

$$
\mu_{R_{j}}(\text { XTest })=\prod_{i=1}^{D} \mu_{R_{j}}\left(\text { XTest }_{i}\right)
$$

is degree of XTest belonged to Rule $R_{j}$ whereas, in the other, Rules is gathered to same group and classification decision is given by:

$$
X \text { Test } \in \text { Class } j^{*} \text { if } j^{*}=\arg \max _{j}\left(\sum_{R_{j} \in \text { Class }} \mu_{R_{j}}(X T e s t) \times C F_{j}\right)
$$

Beside, other authors also have different approaches on fuzzy rules classification system. For example Angelov [23] proposed online learning procedure through two algorithms namely eClass0 and eClass 1 in which rules are adaptive with online mechanism. Wang [7] introduced a new refinement scheme for rules based on the maximization of fuzzy entropy on training set in which he guided to solve quadratic programming. This approach benefits in improvement of generalization of rules as well as overcoming the overfitting of refinement scheme. Ishibuchi [10, 11] improved performance of fuzzy classification system based on rules by applying Genetic Algorithm (GA) approach with single, two and three-objective. By the same approach, Kim [24] also proposed new fitness function for GA and several new XOR membership functions. On the other hand, Verikas [16] limited classifier structure by using SOM tree algorithm and then applied GA to specify input variables for each fuzzy rule.

\section{Proposed Framework}

The most important distinctions between ours and the previous works are we exploit and generate fuzzy rules on metadata to form a classification framework. We apply 4 steps of constructing fuzzy rules system by firstly, Stacking algorithm is applied on training set though base classifiers to generate meta-data (also called Level1 data). After that, fuzzy rules are generated based on predefined membership functions and each component of Level1. Here we select rules from rule set based on both confidence and support of rule that are defined by:

$$
\begin{gathered}
c\left(L_{i} \rightarrow \text { class } j\right)=\frac{\sum_{X \in \text { class } j} \mu_{L_{i}}(X)}{\sum_{X \in \text { Training Set }} \mu_{L_{i}}(X)} \\
s\left(L_{i} \rightarrow \text { class } j\right)=\frac{\sum_{X \in C_{j}} \mu_{L_{i}}(X)}{N}
\end{gathered}
$$

where $\mathrm{N}$ is number of observation in training set. 

16 July 2014

Each rule $L_{i}$ is grouped in same class and its support (5) and confidence (6) are multiple (called rank of a rule) and sort in descending order. We choose the rules in each class with highest rank's value. Finally, all retained rules are saved in a rule set database (Figure 2).

For an unlabeled observation, it is classified by model generated by training set and base classifiers to generate its Level1 data. All rules on rule set employ that data as input and then label is predicted by Single Winner or Vote strategy (3) and (4).

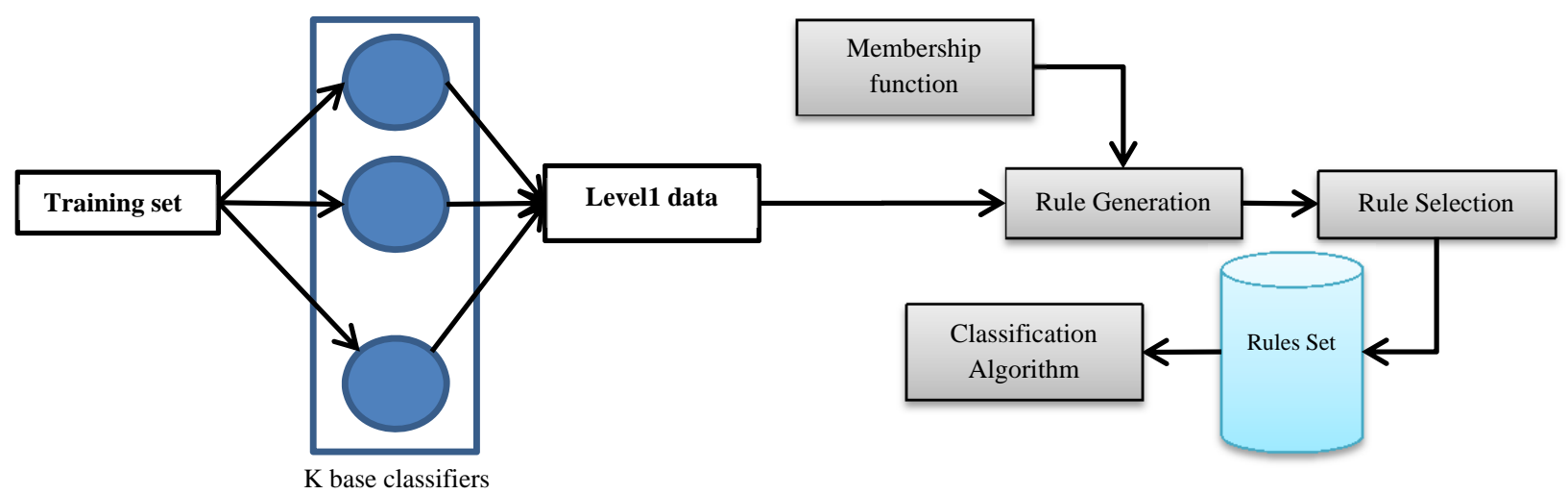

Figure 2. Proposed framework

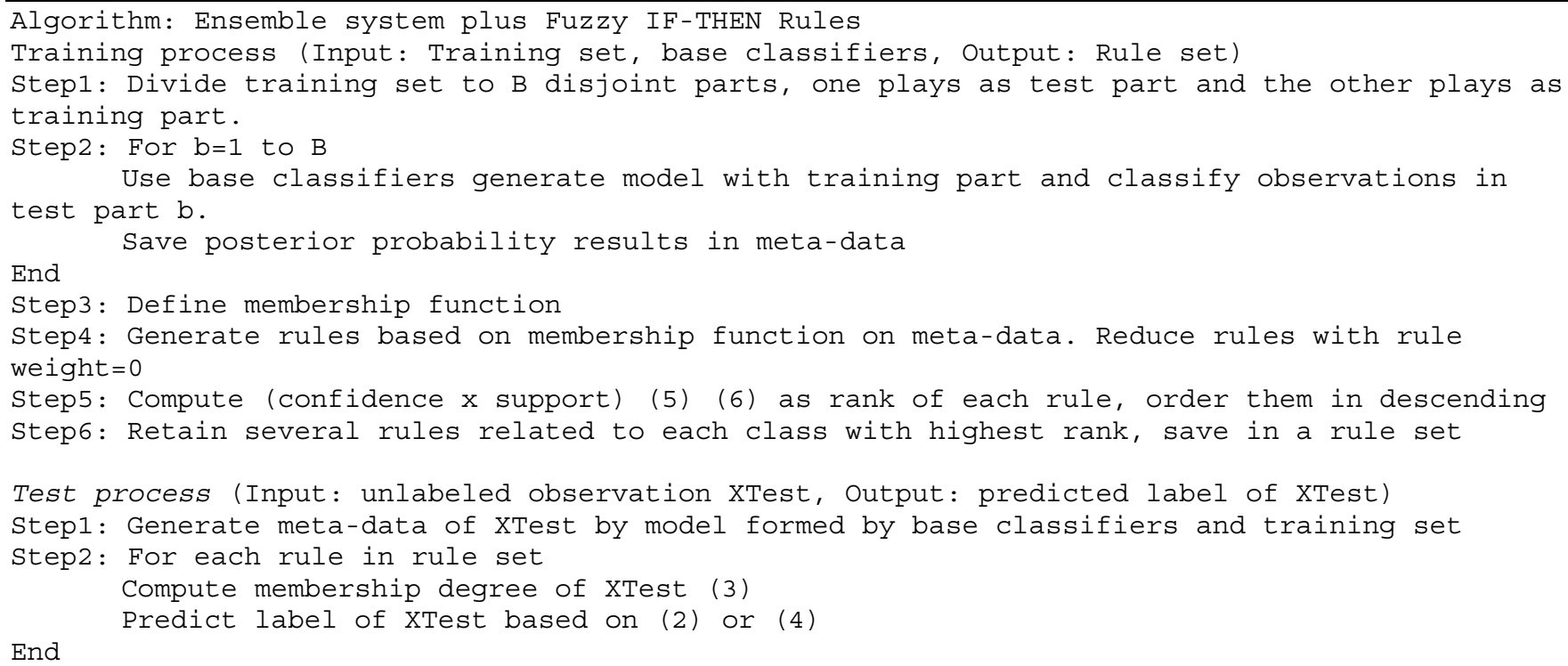

\section{Experimental Results}

Several experiments are fulfilled based on six popular datasets downloaded from UCI Machine Learning Repository (http://archive.ics.uci.edu/ml/datasets.html). Information of these datasets is listed in Table 1 . Here we limit using only three attributes and select only six membership functions (L0, L11, L12, L13, L14, and L15) to generate rules. The goal of this choice is reducing the number of rules to improve the performance of framework. We also choose three traditional classifiers namely Linear Discriminant Analysis (LDA), Naïve Bayes, K Nearest Neighbor (with K set to 5) by the reason is that they all have different approaches so diversity of meta-data is ensured. It is noted that we only have single dataset so to create training set and test set in separation, 10-fold cross validate (CV) technique is used. To ensure objective of test process, we try to run CV produce 10 times so finally we will have 100 test results. 
2014 International Conference on Machine Learning and Cybernetics, ICMLC2014, Lanzhou, China, 1316 July 2014

In comparisons, firstly, we run six fixed combining rules because it is simple and popular in combining classifiers task (Table 2). We also evaluate several state-of-art combining algorithms namely Decision Template, MLR and SCANN (Table 3). Besides, results of twelve fuzzy rules approaches published previously are reported in Table 4. We note that SCANN cannot run on Fertility dataset because its Indicator Matrix is singular [4].

It is interesting to note that our framework is competitive with selected best results from fixed combining rules (1 win and 1 loss), slightly better than Decision Template (2 wins and 0 loss), MLR (2 wins and 1 loss) and SCANN (1 win and 0 loss) (we base on paired t-test with $\alpha$ is set to 0.05). Moreover, our model beats methods of Ishibuchi [8] on Sonar (20.67\% vs. 24.06\%); Soua [22] on Sonar (20.67\% vs. 28.37\% and 30.29\%); Wang weight refinement [17] on Sonar (20.67\% vs. 29\%), Iris (3.3\% vs. 7\%) and Pima (24.6\% vs. 31\%); Verikas [16] on Pima (24.6\% vs. 26.77\%), Gonzalez [22] on Iris (3.3\% vs. 4.3\%); Mansoori [14] on both Pima (24.6\% vs. 26.92\%) and Sonar (20.67\% vs. 24.8\%), Angelov eClass0 [23] on Sonar (20.67\% vs. 29.24\%) and Pima (24.6\% vs. 30.7\%); Angelov eClass1 [23] on Sonar (20.67\% vs. 23.43\%). Those results demonstrate advantage of our model.

There are several cases that our model is worse than other approaches, for instance, Kim [24] on Bupa (33.91\% vs. 29.31\%), Iris (3.3\% vs. 2.01\%) and Pima (24.6\% vs. 23.1\%); Angelov eClass1 [23] on Pima (24.6\% vs. 23.3\%) and Ishibuchi [11] on Iris (3.3\% vs. 2\%). It is worth to consider that several methods like [11] and [24] use GA approach to improve performance of fuzzy rules system whereas here we only use traditional approach for fuzzy rule with some limitation on number of attributes and membership function to generate rule.

TABLE 1: UCI DATASETS IN OUR EXPERIMENT

\begin{tabular}{lccc}
\hline File Name & \# of Attributes & \# of Classes & \\
\hline Bupa & 6 & 2 & \# of observations \\
\hline Fertility & 9 & 2 & 345 \\
\hline Sonar & 60 & 2 & 100 \\
\hline Iris & 4 & 3 & 208 \\
\hline Artificial & 10 & 2 & 150 \\
\hline Pima & 6 & 2 & 700 \\
\hline
\end{tabular}

TABLE 2: CLASSIFICATION ERROR RATE ON UCI DATASETS BY FIXED COMBINING RULES

\begin{tabular}{|c|c|c|c|c|c|c|c|c|c|c|c|c|}
\hline \multirow{2}{*}{ File Name } & \multicolumn{2}{|c|}{ Sum } & \multicolumn{2}{|c|}{ Product } & \multicolumn{2}{|c|}{ Max } & \multicolumn{2}{|c|}{ Min } & \multicolumn{2}{|c|}{ Median } & \multicolumn{2}{|c|}{ Vote } \\
\hline & Mean & Variance & Mean & Variance & Mean & Variance & Mean & Variance & Mean & Variance & Mean & Variance \\
\hline Bupa & 0.3028 & $4.26 \mathrm{E}-03$ & 0.3021 & $4.12 \mathrm{E}-03$ & 0.2986 & $4.15 \mathrm{E}-03$ & 0.2970 & $4.89 \mathrm{E}-03$ & 0.3428 & $4.46 \mathrm{E}-03$ & 0.3429 & 4.04E-03 \\
\hline Fertility & 0.129 & $2.46 \mathrm{E}-03$ & 0.129 & $2.26 \mathrm{E}-03$ & 0.127 & $1.97 \mathrm{E}-03$ & 0.128 & $2.02 \mathrm{E}-03$ & 0.133 & 2.81E-03 & 0.131 & $2.34 \mathrm{E}-03$ \\
\hline Sonar & 0.2259 & 9.55E-03 & 0.2285 & $9.81 \mathrm{E}-03$ & 0.2260 & $7.01 \mathrm{E}-03$ & 0.2298 & $9.32 \mathrm{E}-03$ & 0.2104 & $1.00 \mathrm{E}-02$ & 0.2079 & $8.16 \mathrm{E}-03$ \\
\hline Iris & 0.0387 & 2.59E-03 & 0.0407 & 2.39E-03 & 0.0440 & 3.13E-03 & 0.0413 & $2.56 \mathrm{E}-03$ & 0.0333 & $1.64 \mathrm{E}-03$ & 0.0327 & 1.73E-03 \\
\hline Artificial & 0.2230 & $2.06 \mathrm{E}-03$ & 0.2193 & $2.05 \mathrm{E}-03$ & 0.2450 & $2.57 \mathrm{E}-03$ & 0.2453 & 2.90E-03 & 0.3089 & $1.36 \mathrm{E}-03$ & 0.3073 & 1.03E-03 \\
\hline Pima & 0.2405 & $1.62 \mathrm{E}-03$ & 0.2419 & 1.63E-03 & 0.2411 & $1.69 \mathrm{E}-03$ & 0.2449 & $2.02 \mathrm{E}-03$ & 0.2376 & $1.69 \mathrm{E}-03$ & 0.2365 & $2.10 \mathrm{E}-03$ \\
\hline
\end{tabular}

TABLE 3: CLASSIFICATION ERROR RATE ON UCI DATASETS BY OTHER COMBINING ALGORITHMS

\begin{tabular}{|c|c|c|c|c|c|c|c|c|c|c|}
\hline \multirow{2}{*}{ File Name } & \multicolumn{2}{|c|}{ Decision Template } & \multicolumn{2}{|c|}{ MLR } & \multicolumn{2}{|c|}{ SCANN } & \multicolumn{2}{|c|}{ Select best from fixed combining rules } & \multicolumn{2}{|c|}{ Proposed Method } \\
\hline & Mean & Variance & Mean & Variance & Mean & Variance & Mean & Variance & Mean & Variance \\
\hline Bupa & 0.3348 & $7.10 \mathrm{E}-03$ & 0.3033 & $4.70 \mathrm{E}-03$ & 0.3304 & $4.29 \mathrm{E}-03$ & 0.2986 & $4.15 \mathrm{E}-03$ & 0.3391 & $6.02 \mathrm{E}-03$ \\
\hline Fertility & 0.4520 & $3.41 \mathrm{E}-02$ & 0.1250 & $2.28 \mathrm{E}-03$ & $\mathrm{x}$ & $\mathrm{x}$ & 0.127 & $1.97 \mathrm{E}-03$ & 0.1100 & $4.90 \mathrm{E}-03$ \\
\hline Sonar & 0.2129 & $8.80 \mathrm{E}-03$ & 0.1974 & $7.20 \mathrm{E}-03$ & 0.2128 & $8.01 \mathrm{E}-03$ & 0.2079 & $8.16 \mathrm{E}-03$ & 0.2067 & $7.50 \mathrm{E}-03$ \\
\hline Iris & 0.040 & $2.50 \mathrm{E}-03$ & 0.0220 & $1.87 \mathrm{E}-03$ & 0.0320 & $2.00 \mathrm{E}-03$ & 0.0327 & $1.73 \mathrm{E}-03$ & 0.0330 & $2.89 \mathrm{E}-03$ \\
\hline Artificial & 0.2433 & $1.60 \mathrm{E}-03$ & 0.2426 & $2.20 \mathrm{E}-03$ & 0.2374 & $2.12 \mathrm{E}-03$ & 0.2193 & $2.05 \mathrm{E}-03$ & 0.2257 & $2.02 \mathrm{E}-03$ \\
\hline Pima & 0.2482 & $2.00 \mathrm{E}-03$ & 0.2432 & $2.30 \mathrm{E}-03$ & 0.2384 & $2.06 \mathrm{E}-03$ & 0.2365 & $2.10 \mathrm{E}-03$ & 0.2460 & $2.89 \mathrm{E}-03$ \\
\hline
\end{tabular}

TABLE 4: CLASSIFICATION ERROR RATE ON UCI DATASETS BY SEVERAL FUZZY RULES APPROACHES

\begin{tabular}{|c|c|c|c|c|c|c|}
\hline & $\begin{array}{c}\text { Ishibuchi method } \\
{[8,11]} \\
\end{array}$ & $\begin{array}{c}\text { Soua method [22] } \\
\text { SIFCO } \\
\end{array}$ & $\begin{array}{c}\text { Soua method [22] SIFCO- } \\
\text { PAF }\end{array}$ & $\begin{array}{c}\text { Wang method [17] } \\
\text { refinement }\end{array}$ & $\begin{array}{c}\text { Wang method [17] weight } \\
\text { refinement }\end{array}$ & $\begin{array}{c}\text { Verikas method } \\
{[16]}\end{array}$ \\
\hline Bupa & $\mathrm{X}$ & $x$ & $\mathrm{x}$ & $\mathrm{x}$ & $\mathrm{x}$ & $x$ \\
\hline Sonar & 0.2406 & 0.2837 & 0.3029 & 0.2 & 0.29 & $\mathrm{x}$ \\
\hline Iris & 0.02 & 0.033 & 0.0267 & 0.06 & 0.07 & $\mathrm{x}$ \\
\hline Pima & $\mathrm{x}$ & $\mathrm{x}$ & $\mathrm{x}$ & 0.25 & 0.31 & 0.2677 \\
\hline
\end{tabular}




\begin{tabular}{cccccc}
\hline Kim method [24] & $\begin{array}{c}\text { Gonzalez method [20] } \\
\text { SLAVE 1 }\end{array}$ & $\begin{array}{c}\text { Gonzalez method [20] } \\
\text { SLAVE 2 }\end{array}$ & $\begin{array}{c}\text { Mansoori method [14] } \\
\text { SGERD }\end{array}$ & $\begin{array}{c}\text { Angelov method [23] } \\
\text { eClass1(offline) }\end{array}$ & $\begin{array}{c}\text { Angelov method [23] } \\
\text { eClass0(offline) }\end{array}$ \\
\hline Bupa & 0.2931 & $\mathrm{x}$ & $\mathrm{x}$ & $\mathrm{x}$ & $\mathrm{x}$ \\
\hline Sonar & 0.2248 & $\mathrm{x}$ & $\mathrm{x}$ & 0.2480 & 0.2343 \\
\hline Iris & 0.0201 & 0.043 & 0.043 & 0.036 & $\mathrm{x}$ \\
\hline Pima & 0.2310 & $\mathrm{x}$ & $\mathrm{x}$ & 0.2692 & $\mathrm{x}$ \\
\hline
\end{tabular}

\section{Conclusion and Future Development}

In this paper, we have introduced a new framework by integrating fuzzy IF-THEN rules with ensemble system. Experimental results on 6 popular UCI datasets demonstrate outstanding of our framework compared with state-of-art combining classifiers algorithms as well as other fuzzy rule approaches. Moreover, our model is significantly comprehensive since with meaning of each rule, practitioners can understand the mechanism of classification system. Besides, another benefit of the proposed model is suitable with high dimensional data. Specifically, dimension of Level1 data is equal to number of classifiers multiple with number of classes. For the system with fewer classifiers to solve for example binary classification, Level1 usually has lower dimensions; as a result, it is considerably less complexity to apply rules to Level1 data than to Level0. Finally, our model is convenience and effectiveness in implementation. Level1 is uniformity, real-data type compared with original data which is sometimes very complicated. In addition, Level1 can be viewed as scaled result in $[0,1]$ where observations belong same class have approximately equal posterior probability and hence may be located nearly in the coordination system so it is hoped that it will have more discriminant ability than Level0.

In the near future, we are going to develop this model by studying deeply on membership functions for meta-data which is more suitable than classical one. Moreover, feature selection method is also considered to apply so as to improve performance and effectiveness of the framework.

\section{References}

[1] L. I. Kuncheva, James C. Bezdek and Robert P. W. Duin, "Decision Templates for Multi Classifier Fusion: An Experimental Comparison”, Pattern Recognition, Vol. 34, No. 2, pp. 299-314, 2001.

[2] Kai Ming Ting, Ian H. Witten, "Issues in Stacked Generation”, Journal of Artificial In Intelligence Research, Vol. 10, pp. 271-289, 1999.

[3] Josef Kittler, Mohamad Hatef, Robert P. W. Duin and Jiri Matas, “On Combining Classifiers”, IEEE Transactions on Pattern Analysis and Machine Intelligence, Vol. 20, No. 3, March 1998.

[4] Christopher Merz, “Using Correspondence Analysis to Combine Classifiers”, Machine Learning, Vol. 36, pp. 33-58, 1999.

[5] Mehmet Umut Sen, Hakan Erdogan, "Linear classifier combination and selection using group sparse regularization and hinge loss”, Pattern Recognition Letters 34, pp. 265-274, 2013.

[6] Manuel Valenzuela and Rendon, "The fuzzy classifier system a classifier system for continuously varying variables", Proceeding $4^{\text {th }}$ ICGA, pp. 346-353, 1991.

[7] Hisao Ishibuchi and Takashi Yamamoto, "Fuzzy rule selection by multi-objective genetic local search algorithms and rule evaluation measures in data mining”, Fuzzy Sets and Systems, Vol. 141, pp. 59-88, 2004.

[8] Hisao Ishibuchi and Tanashi Yamamoto, "Comparison of Heuristic Criteria for Fuzzy Rule Selection in Classification Problems”, Fuzzy Optimization and Decision Making, Vol. 3, pp. 119-139, 2004.

[9] Hisao Ishibuchi, Tomoharu Nakashima and Takehiko Morisawa, "Voting in fuzzy rule-based systems for pattern classification problems”, Fuzzy Sets and Systems, Vol. 103, pp. 223-238, 1999.

[10] Hisao Ishibuchi, Tadahiko Murata and I. B. Turksen, "Single-objective and two-objective genetic algorithms for selecting linguistic for pattern classification problems”, Fuzzy Sets and Systems, Vol. 89, pp. 135-150, 1997.

[11] Hisao Ishibuchi, Tomoharu Nakashima and Tadahiko Murata, "Three-objective genetics-based machine learning for linguistic rule extraction”, Information Sciences, Vol. 136, pp. 109-133, 2001.

[12] Tadahiko Murata, Hisao Ishibuchi, Tomoharu Nakashima and Mitsuo Gen, "Fuzzy partition and input selection by genetic algorithms for designing fuzzy rule-based classification systems”, 7th International Conference, EP98 San Diego, California, USA, March 25-27, 1998.

[13] Eghbal G. Mansoori, Mansoor J. Zolghadri and Seraj D. Katebi, “A weighting function for improving fuzzy classification systems performance”, Fuzzy Sets and Systems, Vol. 158, pp. 583-591, 2007. 
2014 International Conference on Machine Learning and Cybernetics, ICMLC2014, Lanzhou, China, 1316 July 2014

[14] Eghbal G. Mansoori, Mansoor J. Zolghadri and Seraj D. Katebi, "SGERD: A Steady-State Genetic Algorithm for Extracting Fuzzy Classification Rules From Data”, IEEE Transactions on Fuzzy Systems, Vol. 16, No. 4, Aug 2008.

[15] Jorge Casillas, Oscar Cordón, María José del Jesus and Francisco Herrera, “Genetic Tuning of Fuzzy Rule Deep Structures Preserving Interpretability and Its Interaction with Fuzzy Rule Set Reduction”, IEEE Transactions on Fuzzy Systems, Vol. 13, No. 1, Feb 2005.

[16] Antanas Verikas, Jonas Guzaitis, Adas Gelzinis and Marija Bacauskiene, “A general framework for designing a fuzzy rule-based classifier”, Knowledge and Information Systems, Vol. 29, No. 1, pp. 203-221, October 2011.

[17] Xi-Zhao Wang and Chun-Ru Dong, “Improving Generalization of Fuzzy IF-THEN Rules by Maximizing Fuzzy Entropy”, IEEE Transactions on Fuzzy Systems, Vol. 17, No. 3, June 2009.

[18] M. Zolghadri Jahromi and M. Taheri, “A proposed method for learning rule weights in fuzzy rule-based classification systems”, Fuzzy Sets and Systems, Vol. 159, pp. 449-459, 2008.

[19] Jesús Alcalá-Fdez, Rafael Alcalá, María José Gacto and Francisco Herrera, "Learning the membership function contexts for mining fuzzy association rules by using genetic algorithms”, Fuzzy Sets and Systems, Vol. 160, No. 7, pp. 905-921, April 2009.

[20] Antonio Gonzalez and Raúl Pérez, “SLAVE: A Genetic Learning System Based on an Iterative Approach”, IEEE Transactions on Fuzzy Systems, Vol. 7, No. 2, pp. 176-191, 1999.

[21] J. L. Castro, J. J. Castro-Schez and J. M. Zurita, “Use of a fuzzy machine learning technique in the knowledge acquisition process”, Fuzzy Sets and Systems, Vol. 123, pp. 307-320, 2001.

[22] Basma Soua, Amel Borgi and Moncef Tagina, “An ensemble method for fuzzy rule-based classification systems”, Knowledge and Information Systems, Vol. 36, No. 2, pp. 385-410, August 2013.

[23] Plamen P. Angelov and Xiaowei Zhou, "Evolving Fuzzy-Rule-Based Classifiers From Data Streams”, IEEE Transactions on Fuzzy Systems, Vol. 16, No. 6, Dec 2008.

[24] Myung Won Kim and Joung Woo Ryu, “Optimized Fuzzy Classification Using Genetic Algorithm”, Second International Conference, FSKD 2005, Changsha, China, August 27-29, 2005.

[25] Lior Rokach, Taxonomy for characterizing ensemble methods in classification tasks: A review and annotated bibliography, Journal of Computational Statistics \& Data Analysis, Vol. 53, Issue 12, pp. 4046 - 4072, October, 2009.

[26] O. Cordon, F. Gomide, F. Herrera, F. Hoffmann and L. Magdalena, "Ten years of genetic fuzzy systems: Current framework and new trends”, Fuzzy Sets and Systems, Vol. 141, No. 1, pp. 5-31, 2004. 